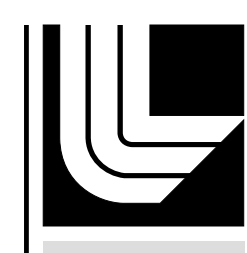

LAWRENCE LIVERM ORE N A TIO NAL LABORATORY

\title{
Plasma-Accelerated Flyer-Plates for Equation of State Studies
}

D. E. Fratanduono, R. F. Smith, T. R. Boehly, J. H. Eggert, D. G. Braun, G. W. Collins

February 23, 2012

Plasma-Accelerated Flyer Plates for Equation of State Studies 
This document was prepared as an account of work sponsored by an agency of the United States government. Neither the United States government nor Lawrence Livermore National Security, LLC, nor any of their employees makes any warranty, expressed or implied, or assumes any legal liability or responsibility for the accuracy, completeness, or usefulness of any information, apparatus, product, or process disclosed, or represents that its use would not infringe privately owned rights. Reference herein to any specific commercial product, process, or service by trade name, trademark, manufacturer, or otherwise does not necessarily constitute or imply its endorsement, recommendation, or favoring by the United States government or Lawrence Livermore National Security, LLC. The views and opinions of authors expressed herein do not necessarily state or reflect those of the United States government or Lawrence Livermore National Security, LLC, and shall not be used for advertising or product endorsement purposes. 


\title{
Plasma-Accelerated Flyer-Plates for Equation of State Studies
}

D. E. Fratanduono ${ }^{1}$, R.F. Smith ${ }^{1}$, T.R. Boehly ${ }^{2}$, J. H. Eggert ${ }^{1}$, D. G. Braun ${ }^{1}$, G. W. Collins ${ }^{1}$

\author{
${ }^{1}$ Lawrence Livermore National Laboratory, Livermore, CA 94550 \\ ${ }^{2}$ Laboratory for Laser Energetic, University of Rochester, 250 East River Road, \\ Rochester, NY 14623-1299
}

We report on a new technique to accelerate flyer-plates to high velocities $(\sim 5 \mathrm{~km} / \mathrm{s})$. In this work, a strong shock is created through direct laser ablation of a thin polyimide foil. Subsequent shock breakout of that foil results in the generation of a plasma characterized by a smoothly increasing density gradient and a strong forward momentum. Stagnation of this plasma onto an aluminum foil and the resultant momentum transfer accelerates a thin aluminum flyer-plate. The aluminum flyer-plate is then accelerated to a peak velocity of $\sim 5 \mathrm{~km} / \mathrm{s}$ before impact with a transparent lithium fluoride $(\mathrm{LiF})$ window. Simulations of the stagnating plasma ramp compression and wave reverberations within the flyer-plate suggest that the temperature at the flyer-plate impact surface is elevated by less than 50 ${ }^{\circ} \mathrm{C}$. Optical velocimetry was used to measure the flyer-plate velocity and impact conditions enabling the shocked refractive index of $\mathrm{LiF}$ to be determined. The results presented here are in agreement with conventional flyer-plate measurements validating the use of plasma-accelerated flyer-plates for equation of state (EOS) and impact studies.

\section{INTRODUCTION}

The dynamic response of materials at high pressure is central to the development of high energy density physics. ${ }^{1}$ Modern dynamic high energy density drivers (i.e. two- 
stage light gas-guns, ${ }^{2,3}$ pulsed-power machines ${ }^{4}$, high-power lasers ${ }^{5,6}$ ) are capable of producing high pressure ( $>100 \mathrm{GPa}$ ) shock compression over significantly different spatial and temporal scales. Gas-gun facilities and pulse-powered devices launch flyerplates that generate strong shocks through collisions, while laser facilities generally produce high-pressure shocks through laser ablation. Laser facilities can produce significantly higher pressures than other platforms, but there are only a few instances of absolute Hugoniot equation of state (EOS) measurements. ${ }^{7,8}$ Laser facilities have relied primarily upon standards to perform EOS studies ${ }^{9,10}$ and the ability to accelerate lowtemperature flyer-plates using laser drivers would enable absolute EOS measurements to be made by symmetric impact.

Flyer-plates are a common experimental tool to perform EOS studies. ${ }^{2-4,11-14}$ The success of those studies relies heavily upon the condition of the flyer-plate at impact. An ideal flyer-plate is able to achieve high velocities, is planar at impact, of substantial thickness to prevent wave reverberations and of a large cross sectional area to mitigate edge effects. Most importantly, the thermodynamic state of the flyer-plate must be uniform and well characterized at impact. Gas-gun facilities have long produced ideal flyer plates. Pulsed-power machines have also achieved excellent results, but laser accelerated flyer-plates have struggled to meet the necessary conditions for useful flyerplates. We describe here a new laser-driven flyer plate that meets the required conditions for shock-compression experiments.

A variety of other laser-driven flyer-plate techniques have been demonstrated. ${ }^{11-14}$ Laser-accelerated flyer-plates, 20-250 $\mu \mathrm{m}$ thick, have been launched through confined ${ }^{11}$ and unconfined ${ }^{12-14}$ laser ablation. These methods are beneficial because there is little 
momentum transfer to the target making the flyer plates easily containable and laser facilities have a high shot rate reducing the average time per experiment. While there laser-accelerated flyer plates demonstrate the great potential for laser-based techniques, there remain substantial experimental difficulties with the state of the flyer-plate at impact. Confined laser ablation launch thin disks from transparent substrates. ${ }^{11}$ The substrate is coated with metallic layers (typically aluminum) that absorb the laser energy. The metallic layer vaporizes, launching a flyer-plate that is limited to $\sim 0.5 \mathrm{~km} / \mathrm{s}$ by substrate absorption and ablation at high laser intensities. In unconfined laser ablation, impedance mismatching and shock reverberations are exploited to accelerate multilayered flyers. ${ }^{12-14}$ The initial shock elevates the temperature of the flyer and the subsequent reverberations continue to raise the temperature. Higher velocities in this method lead to greater entropy production within the sample, significantly raising the temperature of the flyer-plate. The unknown temperature state of the flyer at impact contributes significantly to the uncertainties in impedance matching, undesirable for EOS studies.

In this paper, we improve upon the ability to accelerate flyer-plates using high power lasers. We demonstrate a new flyer-plate technique that is driven by plasma loading produced by the expansion of shock-compressed material. A thin flyer-plate $(\sim 20 \mu \mathrm{m})$ is ramp-compressed and undergoes subsequent multiple wave reverberations. Each wave reverberation accelerates the flyer-plate to a higher velocity. Ramp compression is ideal for generating high-velocity flyers in that it minimizes the temperature increase of the flyer-plate compared to shock-reverberation techniques. ${ }^{12-14}$ In this work, an aluminum flyer-plate is accelerated and collided with a lithium fluoride 
(LiF) window. The planarity, flyer velocity, low temperature and accuracy of this technique are demonstrated by comparing the shocked refractive index measurements of this study with more conventional techniques. In Section II we describe the experimental technique. The results are presented in Section III and conclusions are contained within a discussion in Section IV.

\section{EXPERIMENTAL TECHNIQUE}

Experiments were conducted at the Lawrence Livermore National Laboratory JANUS laser facility. The ramp-wave-loading (RWL) technique developed by Smith et $a l .{ }^{15}$ was used to accelerate aluminum flyer-plates. The laser-driven RWL target design consists of three foils separated by two vacuum gaps. The target is shown schematically in FIG. 1. One beam of the 527-nm Janus laser delivers a total energy of $\sim 300 \mathrm{~J}$ in a $4 \mathrm{~ns}$ square pulse onto a $125-\mu \mathrm{m}$ thick polyimide $\left[\mathrm{C}_{22} \mathrm{H}_{10} \mathrm{~N}_{2} \mathrm{O}_{2}\right]$ foil generating an ablatively driven shock. Phase plates in the beam generate a uniform $\sim 1 \mathrm{~mm}^{2}$ square focal spot that produces a highly uniform planar region $(\Delta I / I \sim \% 5)$ at the focal plane of the laser. A strong shock, produced by laser ablation, propagates through the polyimide foil and then releases adiabatically when it reaches the free (rear) surface. The polyimide rarefies as it expands across a $230 \mu \mathrm{m}$ gap and then stagnates at the surface of a $20-\mu \mathrm{m}$ aluminum ( $99.9 \%$ pure) foil. This results in a smoothly increasing stress wave (ramp-compression) within the aluminum foil. At later times, the compression waves reverberate within the foil and this process increases the velocity of the flyer-plate as a function of time. The plasma-accelerated flyer plate propagates across a secondary $80-\mu \mathrm{m}$ vacuum gap and collides with a $500-\mu \mathrm{m}$ thick transparent $\mathrm{LiF}$ window producing a shock within that 
window. The LiF window is oriented with the $<100>$ axis along the pressure-loading direction.

The velocity of the aluminum flyer plate and the apparent aluminum/LiF interface particle velocity are measured using a velocity interferometry system for any reflector (VISAR). ${ }^{16-18}$ The VISAR measures velocity as a function of time spatially resolved in 1 dimension over a $1 \mathrm{~mm}$ field of view with a spatial resolution of $50 \mu \mathrm{m}$. Two VISAR channels with different sensitivities are used to discern the $2 \pi$ ambiguities that result from the discontinuous fringe jump produced by the flyer-plate collision with the LiF window. The etalon thicknesses in VISAR channels 1 and 2 were $50.0712 \mathrm{~mm}$ and $20.2315 \mathrm{~mm}$ corresponding to VISAR sensitivities of 0.994436 and $2.46114 \mu \mathrm{m} / \mathrm{ns}$ per fringe shift, respectively. The sweep durations for both cameras are 50 ns. The cameras had a spatial field of view of $1 \mathrm{~mm}$. The flyer-plate velocity is observed to be planar over the central $500-\mu \mathrm{m}$ region. The VISAR data is analyzed in the center of the interferometer record to ensure that the edge effects do not influence the experimental results.

\section{Results}

Velocity profiles of shot 091103 are shown in FIG. 2. The transparent LiF window enables the flyer-plate velocity to be measured prior to collision with the window. At 30 ns the stagnation of the polyimide accelerates the aluminum flyer-plate. A continuous acceleration is observed until $\sim 64 \mathrm{~ns}$ when the aluminum flyer-plate collides with the LiF window generating a discontinuity in the velocity record. After collision, the apparent aluminum/LiF interface velocity is recorded (the true interface

velocity requires a density dependent $\mathrm{LiF}$ refractive index correction). ${ }^{19-22}$ We observe a 
steady shock in the $\mathrm{LiF}$ for $\sim 4$ ns until the aluminum release wave (from the drive side of the target) arrives at the aluminum-LiF interface. The duration of the constant apparent velocity is a direct function of the thickness of the flyer-plate used. The velocity profile indicates that the aluminum flyer-plate is accelerating at the time of impact. Higher velocities are achievable by increasing the $80 \mu \mathrm{m}$ vacuum gap.

LiF shock velocity after impact is determined from the aluminum flyer-plate velocity and impedance matching with the LiF window. ${ }^{23}$ Fits to the principle Hugoniot for aluminum $\left(\mathrm{D}=5.4479+1.324 U_{\text {True }}\right)$ and $\mathrm{LiF}\left(\mathrm{D}=5.13+1.31 U_{\text {True }}\right)$ are used, where $\mathrm{D}$ is the shock velocity and $U_{\text {True }}$ is the true particle velocity. ${ }^{24,25}$ The aluminum and $\mathrm{LiF}$ densities are $2.71 \mathrm{~g} / \mathrm{cc}$ and $2.65 \mathrm{~g} / \mathrm{cc}$, respectively. The true particle velocity in the $\mathrm{LiF}$ window was determined from impedance matching and the principle Hugoniots of aluminum and LiF. The principle Hugoniots used in this study were determined at 298 $\mathrm{K}$ and flyer-plates with temperatures above $298 \mathrm{~K}$ will introduce systematic uncertainties into the analysis.

In these experiments, $\mathrm{LiF}$ is shock compressed to $60 \mathrm{GPa}$ and the window remains transparent. The velocity after collision corresponds to the apparent particle velocity at the aluminum $/ \mathrm{LiF}$ interface as measured through the compressed $\mathrm{LiF}$ window. The apparent velocity differs from the true particle velocity since the VISAR probe beam passes through the shock-compressed material of the window. The compressed material has a higher refractive index, increasing the optical path length and imposing a modified Doppler shift within the reflected VISAR probe beam as recorded in the interferometry record. The LiF shocked refractive index $\left(n_{s}\right)$ is defined as, ${ }^{19}$ 


$$
n_{S}=\frac{U_{A p p}-D n_{0}}{U_{\text {True }}-D},
$$

where $U_{A p p}$ is the apparent velocity observed using VISAR, D is the LiF shock velocity, $n_{o}$ is the unshocked refractive index of the $\mathrm{LiF}$ window and $U_{\text {True }}$ is the true particle velocity. The apparent particle velocity $\left(U_{A p p}\right)$ is measured using VISAR.

The flyer-plate velocity and apparent particle velocity after impact are determined from the VISAR velocity profile in FIG. 2. The flyer-plate velocity and apparent particle velocity at the center of the transition are determined by linearly fitting the velocities in a short interval before and after impact. ${ }^{26}$ Fits were extrapolated to the center of the transition to account for the temporal resolution of VISAR. Errors in these extrapolations are included in the uncertainty analysis. The dominant errors result from the precision of fringe-shift measurements, uncertainties in impedance matching technique and the uncertainty in extracting the flyer-plate and apparent particle velocity at impact. Random errors are propagated through the analysis and added in quadrature. Uncertainty in the principal Hugoniot of aluminum and LiF were significantly less than all other sources and neglected. Results of these experiments are shown in Table I.

\section{DISCUSSION}

The refractive index measurements for shocked LiF are shown in FIG. 3. These results are compared with previous shock refractive index measurements of Wise and Chhabilda, ${ }^{19}$ Lalone $e t ~ a l,{ }^{20}$ and Jensen et al. ${ }^{21}$ These results are in good agreement with those measurements performed using flyer-plates accelerated at gas-gun facilities. The close agreement indicates that plasma-accelerated flyer-plates are adequate to quantitatively study materials at high pressures. The error bars in this study result 
primarily from the precision in fringe-shift measurements. The associated errors could be reduced by using more sensitive etalons or by generating higher flyer-plate velocities (producing a low percent error in the flyer-velocity measurement). Drive planarity did not affect these results. Values were obtained at the center of the field of view where the drive is planar and the refractive index was determined instantaneously (at the point of impact).

In all flyer-plate EOS studies, the temperature of the flyer-plate is of significant concern. One-dimensional hydrodynamic LASNEX $^{27}$ simulations were performed to determine the temperature of the flyer-plate at impact with the LiF window. LASNEX simulation use Livermore equation of state (LEOS) tables 5045 and 130 for polyimide and aluminum, respectively. LEOS tables are derived from the quotidian equation of state (QEOS) ${ }^{28}$ The simulated laser drive is tuned to reproduce the velocity of the aluminum flyer-plate of shot 91103. Comparison of the LASNEX simulated and the VISAR measured flyer-plate velocity is shown in FIG 4. An inherent limitation of the LASNEX hydro-code is the inability to simulate the low-density leading edge of the polyimide release, characterized by a spread in particle velocities and arrival times on the aluminum flyerplate. LASNEX simulates the polyimide as a number of discrete cells, each with a single velocity. As a result, when the first polyimide cell impacts the aluminum it produces a weak shock that abruptly increases the temperature of the flyer-plate as shown in FIG 4 . This is contrary to the experimental results that show a smoothly increasing flyer-plate velocity, indicative of ramp compression. Regardless, these simulations indicate that the temperature of the aluminum flyer-plate at impact is elevated by less than $50{ }^{\circ} \mathrm{C}$ which is an over estimation of the actual flyer-plate temperature due to the addition of shock heating. It is important to note that LASNEX simulations closely predict the actual flyer-plate velocity 
prior to impact with the $\mathrm{LiF}$ window. This indicates that the momentum transfer from the polyimide release to aluminum flyer-plate is well treated.

The LASNEX simulation predicts that the thermal wave generated from the stagnating plasma arrives at the free surface of the aluminum flyer several hundred nanoseconds after stagnation begins. A temperature contour plot is shown in FIG 5. The aluminum flyer-plate corresponds to the Lagrangian depth of 0 to $20 \mu \mathrm{m}$. At $\sim 30 \mathrm{~ns}$ the rarefied polyimide arrives at the aluminum flyer-plate and stagnation begins. A weak shock is generated within the aluminum sample elevating the temperature by $30{ }^{\circ} \mathrm{C}$. Multiple wave reverberations are observed as the temperature equilibrates. The heat conduction from the stagnating $\mathrm{CH}$ and propagation into the aluminum is shown. In these experiments, impact between the aluminum flyer and LiF window occur $\sim 35 \mathrm{~ns}$ after stagnation well before the arrival of the thermal wave. In contrast to these experiments, significantly higher temperatures are achieved in the shock reverberation method. ${ }^{12-14}$ To obtain an aluminum flyer-plate velocity of $5 \mathrm{~km} / \mathrm{s}$ with a polyimide backing, the temperature of the aluminum would exceed $\sim 500{ }^{\circ} \mathrm{C}$. The ramp wave reverberation technique presented here produces significantly lower temperatures and is better suited for EOS studies than previously demonstrated laser-driven flyers.

We have successfully demonstrated the use of plasma-accelerated flyer-plates to perform EOS measurements. VISAR measurements of the flyer-plate and aluminum/LiF interface velocity enabled the shock refractive index of $\mathrm{LiF}$ to be determined. This new technique is a viable process to produce high-pressure shocks using laser-drivers enabling absolute EOS measurements. This work performed under the auspices of the U.S. 
Department of Energy by Lawrence Livermore National Laboratory under Contract DEAC52-07NA27344.

\section{BIBLIOGRAPHY}

1. "Frontiers for Discovery in High Energy Density Physics," prepared for the Office of Science and Technology Policy, National Science and Technology Council Interagency Working Group on the Physics of the Universe and prepared by the National Task Force on High Energy Density Physics (20 July 2004).

2. A. H. Jones, W. M. Isbell, and C. J. Maiden, Journal of Applied Physics 37, 3493 (1966).

3. J.A. Morgan, High Temp. High Pressures 6 (1974).

4. R. B. Spielman, C. Deeney, G. A. Chandler, M. R. Douglas, D. L. Fehl, M. K. Matzen, D. H. McDaniel, T. J. Nash, L. J. Porter, T. W. L. Sanford, J. F. Seamen, W. A. Stygar, K. W. Struve, S. P. Breeze, J. S. McGurn, J. A. Torres, D. M. Zagar, T. L. Gilliland, D. O. Jobe, J. L. McKenney, R. C. Mock, M. Vargas, T. Wagoner, and D. L. Peterson, Phys. Plasmas 5, 2105 (1998).

5. E. I. Moses, Fusion Sci. Technol. 54, 361 (2008).

6. T. R. Boehly, D. L. Brown, R. S. Craxton, R. L. Keck, J. P. Knauer, J. H. Kelly, T. J. Kessler, S. A. Kumpan, S. J. Loucks, S. A. Letzring, F. J. Marshall, R. L. McCrory, S. F. B. Morse, W. Seka, J. M. Soures, and C. P. Verdon, Opt. Commun. 133, 495 (1997).

7. L. B. Da Silva, P. Celliers, G. W. Collins, K. S. Budil, N. C. Holmes, T. W. Barbee Jr, B. A. Hammel, J. D. Kilkenny, R. J. Wallace, M. Ross, R. Cauble, A. $\mathrm{Ng}$, and G. Chiu, Physical Review Letters 78, 483 (1997).

8. A. Benuzzi-Mounaix, M. Koenig, G. Huser, B. Faral, D. Batani, E. Henry, M. Tomasini, B. Marchet, T. A. Hall, M. Boustie, Th De Resseguier, M. Hallouin, F. Guyot, D. Andrault, and Th Charpin, Physics of Plasmas 9, 2466 (2002).

9. D. G. Hicks, T. R. Boehly, P. M. Celliers, J. H. Eggert, E. Vianello, D. D. Meyerhofer, and G. W. Collins, Physics of Plasmas 12, 082702 (2005).

10. M. A. Barrios, D. G. Hicks, T. R. Boehly, D. E. Fratanduono, J. H. Eggert, P. M. Celliers, G. W. Collins, and D. D. Meyerhofer, Physics of Plasmas 17, 056307 (2010).

11. Damian C. Swift, Johnathan G. Niemczura, Dennis L. Paisley, Randall P. Johnson, Sheng-Nian Luo, Thomas E. Tierney, and Iv, Review of Scientific Instruments 76, 093907 (2005).

12. K. Takamatsu, N. Ozaki, K. A. Tanaka, T. Ono, K. Nagai, M. Nakai, T. Watari, A. Sunahara, M. Nakano, T. Kataoka, H. Takenaka, M. Yoshida, K. Kondo, and T. Yamanaka, Physical Review E 67, 056406 (2003).

13. N. Ozaki, Y. Sasatani, K. Kishida, M. Nakano, M. Miyanaga, K. Nagai, K. Nishihara, T. Norimatsu, K. A. Tanaka, Y. Fujimoto, K. Wakabayashi, S. Hattori, T. Tange, K. Kondo, M. Yoshida, N. Kozu, M. Ishiguchi, and H. Takenaka, Journal of Applied Physics 89, 2571 (2001). 
14. Kazuo A. Tanaka, Motohiko Hara, Norimasa Ozaki, Yasufumi Sasatani, Sergei I. Anisimov, Ken-ichi Kondo, Motohiro Nakano, Katsunobu Nishihara, Hisataka Takenaka, Masatake Yoshida, and Kunioki Mima, Physics of Plasmas 7, 676 (2000).

15. R. F. Smith, J. H. Eggert, M. D. Saculla, A. F. Jankowski, M. Bastea, D. G. Hicks, and G. W. Collins, Physical Review Letters 101, 065701 (2008).

16. L. M. Barker and R. E. Hollenbach, J. Appl. Phys. 43, 4669 (1972).

17. P. M. Celliers, G. W. Collins, L. B. Da Silva, D. M. Gold, and R. Cauble, Appl. Phys. Lett. 73, 1320 (1998).

18. P. M. Celliers, D. K. Bradley, G. W. Collins, D. G. Hicks, T. R. Boehly, and W. J. Armstrong, Rev. Sci. Instrum. 75, 4916 (2004).

19. J. L. Wise and L. C. Chhabildas,(NTIS Order No. DE85015505), Sandia National Laboratory, Albuquerque, NM, Report SAND-85-0310C, NTIS Order No. DE85015505 (1985).

20. B. M. LaLone, O. V. Fat'yanov, J. R. Asay, and Y. M. Gupta, J. Appl. Phys. 103, 093505 (2008).

21. B. J. Jensen, D. B. Holtkamp, P. A. Rigg, and D. H. Dolan, J. Appl. Phys. 106, 049901 (2009).

22. D. E. Fratanduono, J. Appl. Phys. 109, 123521 (2011).

23. G. R. Gathers, Selected Topics in Shock Wave Physics and Equation of State Modeling (World Scientific, Singapore, 1994).

24. P. M. Celliers, J. Appl. Phys. 98, 113529 (2005).

25. R. F. Trunin, ed. Experimental Data on Shock Compression and Adiabatic Expansion of Condensed Matter (Russian Federal Nuclear Center-VNIIEF, Sarov, 2001).

26. D. G. Hicks, T. R. Boehly, P. M. Celliers, D. K. Bradley, J. H. Eggert, R. S. McWilliams, R. Jeanloz, and G. W. Collins, Phys. Rev. B 78, 174102 (2008).

27. G. B. Zimmerman and W. L. Kruer, Comments Plasma Phys. Control. Fusion 2, 51 (1975).

28. R. M. More, K. H. Warren, D. A. Young, and G. B. Zimmerman, Physics of Fluids 31, 3059 (1988). 
FIG. 1. Target schematic for indirect-drive accelerated flyer-plate. The Janus laser ablates a $125-\mu \mathrm{m}$ polyimide sample generating a strong shock. Upon breakout from the rear surface strong-shock heating and momentum causes the polyimide to unload as plasma across a $230 \mu \mathrm{m}$ wide vacuum gap. The released polyimide expands as it propagates and eventually stagnates at the aluminum surface. Momentum transfer results in ramp compression and subsequent acceleration of a $20-\mu \mathrm{m}$ thick aluminum flyer plate. The aluminum flyer plate propagates across a vacuum gap and collides with a LiF window. The flyer-plate velocity prior to impact and apparent particle velocity post impact are measured using the VISAR diagnostic. ${ }^{16-18}$

FIG. 2. VISAR velocity profiles for shot 091103 . Prior to $\sim 64 \mathrm{~ns}$ the flyer-plate acceleration is recorded. After the aluminum flyer plate and LiF impact, the apparent interface particle velocity is recorded. The release wave from the aluminum flyer is observed at $\sim 68 \mathrm{~ns}$ marked by a decrease in the observed apparent interface velocity.

FIG. 3. Shocked refractive index measurements of this study (circles) are compared to measurements made by Wise and Chhabilda ${ }^{19}$ [square], Lalone et al. ${ }^{20}$ [triangle] and Jensen et al. ${ }^{21}$ [diamond] at $532 \mathrm{~nm}$.

FIG. 4. Comparison of the measured VISAR aluminum flyer-plate velocity (black) for shot 91103 and the LASNEX simulated velocity profile (black solid line) (left axis). Impact with the LiF at $64 \mathrm{~ns}$ is not included in the LASNEX simulations. LASNEX cannot accurately model the low-density leading edge of the rarefied plasma. This manifests itself as a weak initial shock at $\sim 34 \mathrm{~ns}$. The LASNEX predicated temperature of the aluminum free surface is shown on the right axis (in red). The weak shock initially heats the sample by $\sim 30{ }^{\circ} \mathrm{C}$ and the wave reverberations raise the temperature by an additional $\sim 20{ }^{\circ} \mathrm{C}$ at the time of impact. This represents an upper bound to the flyer-plate temperature due to the addition of shock heating that is not present in the experiment.

FIG. 5. LASNEX simulated temperature contour plot. Stagnation of the polyimide plasma onto the $20 \mu \mathrm{m}$ aluminum flyer-plate is shown. At $\sim 30 \mathrm{~ns}$ polyimide stagnation begins. An initial week shock propagates through the aluminum and arrives at the free surface at $\sim 34 \mathrm{~ns}$. Multiple wave reverberations are observed as the temperature in the aluminum equilibrates. 
TABLE I: Flyer-Plate impact experimental parameters.

\begin{tabular}{lcc}
\hline \hline & Shot 91103 & Shot 91105 \\
\hline Flyer Velocity $(\mu \mathrm{m} / \mathrm{ns})$ & $4.87( \pm 0.03)$ & $5.23( \pm 0.03)$ \\
Apparent Velocity $(\mu \mathrm{m} / \mathrm{ns})$ & $3.17( \pm 0.03)$ & $3.42( \pm 0.03)$ \\
True Velocity $(\mu \mathrm{m} / \mathrm{ns})$ & $2.49( \pm 0.02)$ & $2.68( \pm 0.02)$ \\
Shock Velocity $(\mu \mathrm{m} / \mathrm{ns})$ & $8.39( \pm 0.02)$ & $8.63( \pm 0.02)$ \\
Pressure $(\mathrm{GPa})$ & $55.4( \pm 0.5)$ & $61.1( \pm 0.5)$ \\
Density $(\mathrm{g} / \mathrm{cc})$ & $3.77( \pm 0.01)$ & $3.83( \pm 0.01)$ \\
Refractive Index & $1.444( \pm 0.006)$ & $1.445( \pm 0.006)$ \\
\hline
\end{tabular}

